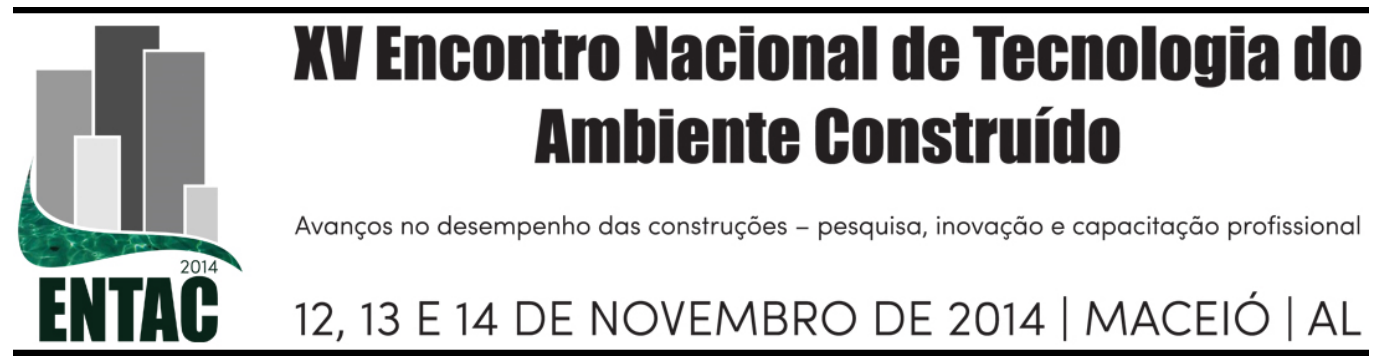

\section{ESTUDO DE OTIMIZAÇÃO DE COBERTURAS RESPONSÍVAS À INSOLAÇÃO ATRAVÉS DA PARAMETRIZAÇÃO}

\author{
CAMPOS, Filipe (1); CELANI, Maria Gabriela (2)
}

(1) Unicamp, e-mail: f_m_campos@yahoo.com.br (2) Unicamp, e-mail: gabi.celani@gmail.com

\begin{abstract}
RESUMO
Nas últimas décadas as tecnologias de CAD/CAM desenvolveram-se, abrindo novas possibilidades para a produção e ensino de arquitetura e urbanismo. Ferramentas como modelagem NURBS, parametrização e prototipagem rápida foram inseridas no cotidiano da produção arquitetônica, e estão mudando a forma de pensá-la. Cada vez mais são usadas superfícies curvas e complexas em grandes construções, como aeroportos e museus, sendo necessário analisar seu desempenho térmico para fins de condicionamento adequado do ar, bem-estar do usuário e redução do consumo energético. Este artigo visa apresentar uma pesquisa em desenvolvimento sobre o uso de um método generativo paramétrico para otimizar a proteção solar de superfícies complexas.
\end{abstract}

Palavras-chave: Parametrização, otimização solar, sistema generativo.

\begin{abstract}
On the last decades the CAD/CAM technologies evolved, allowing new possibilities for the production and teaching of architecture and urban design. Tools such as NURBS modeling, parametric modeling and rapid prototyping were introduced into architecture production, and they are changing design thinking. Curved and complex forms have become more common in large buildings such as airports and museums, and it is necessary to analyze their thermal performance to provide the correct air conditioning, user well-being and reduce the amount of energy spent. This paper describes an undergoing research about the use of a parametric generative method to optimize solar shading in complex surfaces.
\end{abstract}

Keywords: Parameterization, solar optimization, generative systems.

\section{INTRODUÇÃO}

O desenvolvimento das ferramentas computacionais em arquitetura tem permitido projetar e construir edifícios que não seriam possíveis há dez anos (BURRY, 2010). As simulações computacionais, modelagem NURBS, parametrização e tecnologias de prototipagem rápida estão modificando os métodos tradicionais de projeto, não só pela inserção de novos espaços matemáticos e formas (superfícies contínuas ou de múltiplas curvaturas), mas também pelas possibilidades de otimização (seja ambiental, estrutural etc) e uso de parâmetros no desenvolvimento dos projetos, baseando-se em suas relações topológicas (BURRY, 2010).

Cada vez mais são introduzidas na arquitetura formas complexas com múltiplas curvaturas, sendo as tecnologias de simulação importantíssimas, pois permitem automatizar o processo de cálculo, fornecendo rapidez e precisão. Independentemente da complexidade das formas e estruturas, é possível calcular e simular desde a tensão estrutural do edifício até seu ganho térmico anual, prevendo os gastos materiais e energéticos envolvidos. Porém, só quantificar estes aspectos é um uso limitado das 
capacidades computacionais de simulação, sendo de grande importância a otimização do edifício, visando diminuir a energia e materiais gastos em sua construção e operação. Baseando-se em parâmetros de projeto é possível modificá-lo, mantendo suas relações espaciais, buscando atingir um alto desempenho energético e material.

Tendo em vista as questões de desempenho térmico do edifício e bem-estar dos usuários, esta pesquisa baseia-se no estudo do uso da parametrização e simulações computacionais para otimizar o ganho térmico de coberturas. Considerando o ganho térmico como um parâmetro projetual, pode-se modifica-lo e ajustar de acordo com seus valores ideias para a redução do consumo de energia por condicionamento de ar.

Pela modificação de aspectos como implantação, rotação, forma, permeabilidade visual e outros, é possível maximizar ou minimizar o ganho térmico, sendo possível também ajusta-lo de acordo com o necessário, aproximando-se de seu valor ideal. Estes ajustes são feitos com o uso de algoritmos genéticos, que utilizam os parâmetros citados e outros para atingir o valor estabelecido.

Com a ampliação do uso de formas de múltiplas curvaturas na arquitetura, esta pesquisa se foca na otimização deste tipo de edifícios, porém podem ser usados os mesmos princípios em formas ortogonais. Além de criar um sistema de otimização de superfícies, a pesquisa busca demonstrar o uso das ferramentas de parametrização e como estas permitem soluções eficientes e otimizadas em geometrias complexas.

\section{FUNDAMENTAÇÃO}

Como citado na introdução, as inovações dos últimos anos permitiu aos arquitetos estender sua criatividade a espaços geométricos abertos pelos matemáticos dos últimos três séculos (BURRY, 2010), sendo que a introdução de “(...) NURBS (Non-Uniform Rational B-Splines), i.e. curvas e superfícies paramétricas, abriu um universo de formas complexas que eram, até o surgimento das tecnologias de CAD/CAM, muito difíceis de conceber, desenvolver e representar, sem mencionar em produzir.” (KOLAREVIC, 2005, tradução própria).

A complexidade e geometria destas formas não permite a aplicação de regras tipo "ruleof-thumb", como implantação e orientação solar. Com o crescente aumento na demanda de edifícios sustentáveis, o alto desempenho térmico é um aspecto de grande importância a ser levado em conta. Necessitando de analises computacionais para garantir este desempenho, o uso da parametrização é fundamental.

A parametrização em arquitetura se baseia no conceito de automatização do processo de projeto, onde são definidas regras baseadas em parâmetros para criar, analisar e modificar formas e estruturas para atender a diferentes critérios. Estes parâmetros podem variar desde definições geométricas e relações entre elementos espaciais até valores de ganho solar e rigidez estrutural, permitindo otimizar o projeto e criar obras arquitetônicas inteligentes (DIMCIC, 2013). Essa automação permite também a redução do tempo gasto no desenvolvimento do projeto, fornecendo resultados mais precisos.

\section{MATERIAIS E MÉTODOS}

A pesquisa desenvolvida é do tipo experimental e possuí caráter exploratório, inserindose na área de Building Performance Simulation (BPS). Para o desenvolvimento desta, foi utilizado o programa de modelagem Rhinoceros 5RS2, com seus plug-ins Grasshopper (versão 0.9.0072) e Geco (versão 1.0.43.0) e o programa de análise Ecotect Analysis 2011. O plug-in Grasshopper é responsável pela implementação do modelo 
paramétrico, utilizando programação visual, enquanto o plug-in Geco é responsável pela conexão entre o Grasshopper e o Ecotect. Para fins de análise de insolação, foi utilizado o arquivo de dados meteorológicos (WeatherFile) de São Paulo - SP.

A geometria da cobertura foi desenvolvida para ser assimétrica, com dupla curvatura e possuir áreas que seriam sombreadas por sua própria forma. Desde modo, garantiu-se maior heterogenia dos valores de insolação e ganho térmico do que se a cobertura fosse simétrica, ortogonal ou sem auto-sombreamento. Para conseguir otimizar esta cobertura, foram delimitados quatro passos a partir da modelagem desta (Figura 1): subdivisão da superfície, aplicação de padrões à superfície, cálculo inicial de ganho térmico e otimização pelo uso de algoritmos genéticos.

\section{Figura 1 - Sequência de ações para otimização}
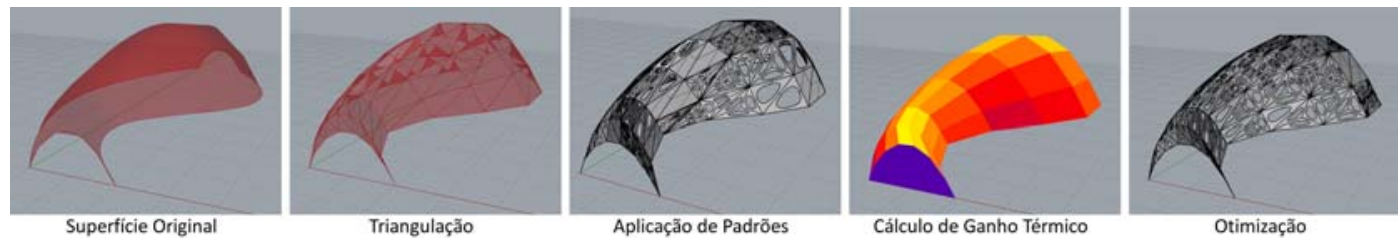

Fonte: própria

\subsection{Triangulação}

Como método de subdivisão, foi escolhida a triangulação da superfície, pois facilita a construção e montagem da estrutura. Além disso, o triângulo é a única forma capaz de definir um plano, tendo um vetor normal definido. Garante-se também que seus vértices pertencem sempre à superfície original, diminuindo a chance de deformações. Existem diversos tipos de triangulações, sendo usada normalmente a triangulação por subdivisão de quadriláteros, por subdivisão de hexágonos ou através da triangulação de Delaunay.

A triangulação de Delaunay foi desenvolvida para evitar erros na triangulação de terrenos, baseando-se na conexão de pontos próximos (BERG, 2008). Pela irregularidade das superfícies complexas este método foi o primeiro a ser testado, além disso, o Grasshopper apresenta o componente de triangulação de Delaunay, facilitando seu uso. Porém, esta forma de triangulação apresentou problemas em suas bordas, criando diagonais e superfícies que não existiam, não sendo adequada à pesquisa.

A triangulação por subdivisão de hexágonos foi levada em conta por permitir maior controle sobre o comprimento das arestas das faces trianguladas, porém sua aplicação baseia-se em algoritmos complexos e sistemas de loopings, buscando uma melhor distribuição dos hexágonos pela superfície. Por não ser a triangulação o objetivo da pesquisa, este método foi excluído do processo.

O método escolhido foi o de triangulação por subdivisão de quadriláteros, que fornece um resultado de subdivisão satisfatório (Figura 2). Foi feito pela divisão da superfície em subsuperfícies isoparamétricas, pelos parâmetros $\mathrm{U}$ e $\mathrm{V}$, fornecendo subsuperfícies quadriláteras, ou seja, com quatro bordas curvas e quatro vértices. Como cada subsuperfície apresenta seus vértices independentemente uma das outras, foi possível ensinar o programa a ler os vértices (0, 1, 2 e 3) na sequencia correta, criando uma diagonal no meio. Cada subsuperfície quadrilátera forneceu dois triângulos, sendo o primeiro gerado pelos vértices 0,1 e 2 e o segundo pelos vértices 0,2 e 3 . 


\section{Figura 2 - Método de triangulação por subdivisão de quadriláteros}
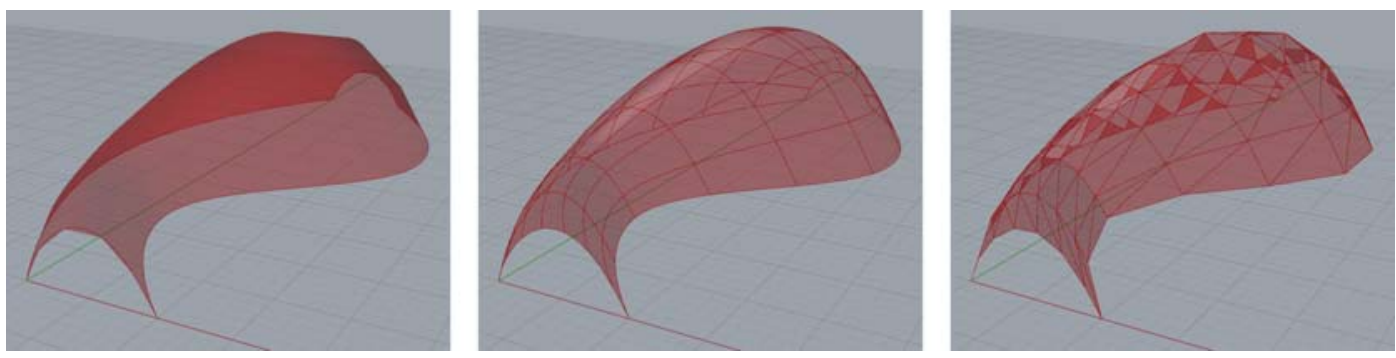

Fonte: própria

A criação das superfícies pelos vértices forneceu superfícies triangulares independentes, facilitando depois o processo de cálculo de área, incidência solar e ganho térmico total. A desvantagem deste método é que, por não se tratar de uma superfície regular, os triângulos podem apresentar dimensões maiores num sentido que no outro, como grande comprimento e pequena largura.

\subsection{Aplicação de padrões}

Definido o método de triangulação, foi necessário aplicar padrões às faces, com o objetivo de criar aberturas controláveis por parâmetros. Essas aberturas (Figura 3) regulam a quantidade de iluminação direta no interior da construção e o ganho térmico, variando-se os materiais usados e suas propriedades de condução térmica. $\mathrm{O}$ Grasshopper permite a aplicação de geometrias em superfícies, utilizando um método de “caixas”, onde se cria um paralelepípedo circunscrito à geometria a ser aplicada e um circunscrito à cada subsuperfície, adaptando a forma original à forma de cada subsuperfície. Porém esse método foi desenvolvido visando geometrias e subsuperfícies quadriláteras.

\section{Figura 3 - Painéis a serem aplicados}
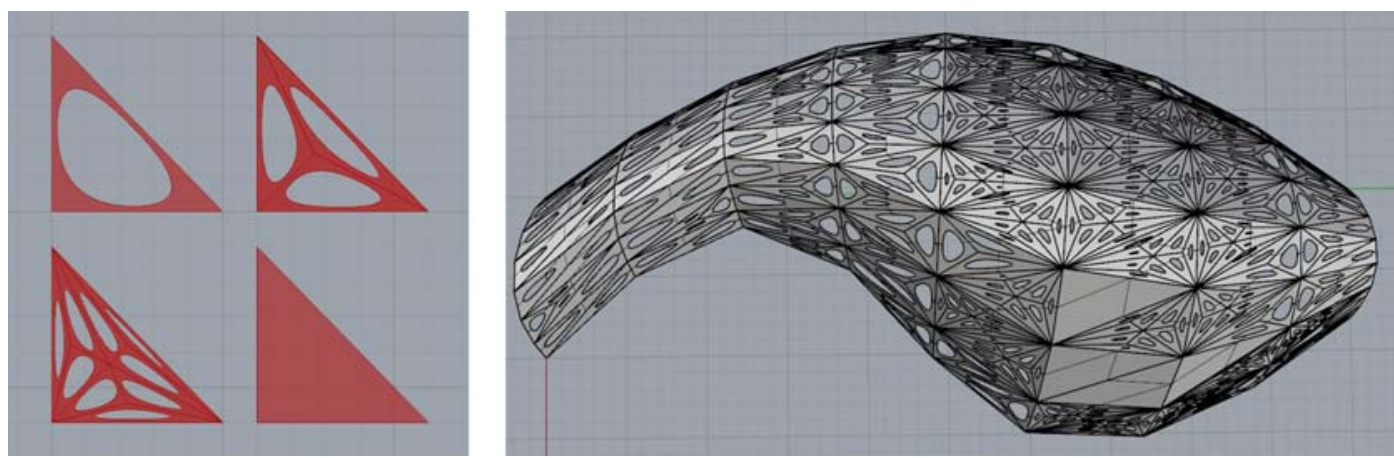

Fonte: própria

Para utilizar com triângulos, foi necessário tratar os padrões e faces trianguladas como quadriláteros. Nesse método, foi necessário desenhar o triângulo-base dos padrões de acordo com a ordem de leitura de vértices da triangulação, alinhando-os.

Foram testados também o uso de padrões parametrizados, baseando-se nas relações topológicas desejadas. Este método apresentou padrões mais bem definidos, com diminuição significativa dos erros, sendo escolhido para os testes de otimização por variação de padrões. 


\subsection{Cálculo de ganho térmico}

Para medir a insolação incidente nas faces foram usadas duas técnicas: a primeira utilizou somente componentes do Grasshopper, já a segunda técnica utilizou a conexão com Ecotect via Geco. Para medição do ganho solar é necessário ter a insolação incidente em cada face, sua área e características do material (transmissão térmica), sendo que, tendo a superfície triangulada e dividida em subsuperfícies, é fácil de se obter a área de cada face.

Para o calculo da insolação incidente, primeiro tentou-se desenvolver em Grasshopper um método de cálculo, utilizando o vetor solar para obter a área virtual da face. O método apresentou diversas limitações, principalmente por não considerar a sombra que cada elemento projetual faria nos demais, como a programação não era capaz de ler essa interação, assumia que todas as faces estavam expostas diretamente ao vetor solar. Além disso, seria necessário obter o vetor solar pelo Geco.

O Geco, por sua conexão com o Ecotect, já permitia o calculo automático da insolação incidente, sendo capaz de relacionar a questão de sombreamento na superfície. Deste modo foi usado o Ecotect para obter a insolação incidente das faces na superfície. $\mathrm{O}$ processo consiste, basicamente, de enviar a geometria do Grasshopper para o Ecotect e solicitar o cálculo de insolação incidente, controlando via Geco as variáveis do cálculo, como período do ano e do dia.

Durante sua utilização, foi necessário prestar atenção em alguns fatores importantes para garantir a leitura e cálculo correto das faces. O primeiro deles é a necessidade de se ter um volume fechado para ser exportado para o Ecotect. No caso de superfícies ou volumes abertos, podem ocorrer problemas no calculo de insolação, por inversão da normal da face e etc,. Nos primeiros testes utilizaram-se apenas superfícies abertas, sendo notados resultados absurdos, como baixíssima insolação de faces expostas ao sol durante quase todo o período do dia. Foi notado então que, criando o volume fechado do ambiente, o calculo é feito do modo correto.

O segundo fator é a forma de exportação do Grasshopper para o Ecotect, pois este só importa formas tipo MESH. Essa forma é difícil de se trabalhar por problemas na forma de identificação de vértices, faces e arestas, sendo estas informações compiladas num modo de leitura diferente do que quando utilizando faces comuns. Durante este processo, o programa reordena as faces para cálculo, sendo que foi necessário reordenar as faces e o resultado das insolações para poder relacionar corretamente as insolações com as áreas.

Para se fazer a reordenação, foi usado como referencia a distância entre o centro das faces MESH e o centro da projeção da superfície no plano, sendo que todos os dados fornecidos pelo Ecotect foram reordenados de acordo com essa relação. Do mesmo modo, reordenaram-se as faces originais e seus dados (como área), tornando possível relacionar a insolação incidente e a face corretamente. Dificilmente duas faces terão a mesma distância do centro em caso de superfície de múltiplas curvaturas, sendo que em nenhum dos testes isso ocorreu. Contudo, é possível que ocorra. Em volume regulares, será mais fácil, sendo necessário colocar o ponto fora do centro e dos eixos de espelhamento.

Com as faces ordenadas, podendo relacionar corretamente a área de cada face com sua insolação, foi possível calcular o ganho térmico solar. Com o calculo pronto, pode-se utilizar o Galápagos, componente do Grasshopper que utiliza algoritmos genéticos, para otimizar o parâmetro desejado. 


\section{RESULTADOS}

A partir de uma superfície modelada em Rhinoceros foram efetuadas analises e modificações quanto a sua orientação (por meio de rotação), sua área de absorção (pela alteração da altura dos vértices dos triângulos) e sua permeabilidade visual (pela aplicação padrões às faces). A superfície cobre uma área de $788 \mathrm{~m}^{2}$, apresentando originalmente um ganho térmico solar de 1484 MWh.

As rotações efetuadas foram feitas rotacionando a superfície original sobre seu centro. A superfície original apresentava orientação sentido norte-sul, sendo que quanto foi efetuada a sua rotação, notou-se que a orientação ideal para maximizar o ganho térmico era de $12^{\circ}$, sendo o resultado próximo do da superfície original, com ganho térmico de 1485 MWh. Já para minimizar o ganho térmico, obteve-se uma rotação de $95^{\circ}$, com um ganho térmico de 1470 MWh (Figura 4).

Figura 4 - Otimização do ganho térmico por rotação (vistas em planta)


Fonte: própria

Como se pode notar, a diferença de valores entre a superfície original e as modificadas não foi significativa, sendo necessário testar outros métodos de modificação da superfície para sua otimização. Em seguida, foi testada então a alteração da área de absorção.

Para alterar a área de absorção foi necessário modificar formalmente a cobertura. Neste caso, foi decidido manter-se área protegida pela cobertura, mantendo as coordenadas $\mathrm{X}$ e Y dos vértices dos triângulos da superfície. Modificando a coordenada $\mathrm{Z}$, a área protegida mantem-se a mesma, porém a área superficial da cobertura pode ser aumentada ou reduzida. Por exemplo, um triângulo de vértices A $(2,0,4), \mathrm{B}(0,0,3)$ e C $(1,1,2.5)$ possuí $1.5 \mathrm{~m}^{2}$ e protege uma área de $1 \mathrm{~m}^{2}$, ao se aumentar o valor da coordenada Z (altura do ponto) em 25\%, o novo triângulo protegerá a mesma área $\left(1 \mathrm{~m}^{2}\right)$, porém a área de sua superfície será de $1.72 \mathrm{~m}^{2}$ (14,6\% maior).

Este método permite modificar a forma da cobertura para adequar-se ao ganho térmico desejado, sendo que é possível também modificar os pontos quanto às coordenadas $\mathrm{X} \mathrm{e}$ Y. Como a modificação na altura dos pontos afeta diretamente a área das faces, o valor do ganho térmico também será alterado. Além da área, este método também pode modificar a inclinação de algumas faces, alterando o valor do ganho térmico.

Com o aumento em $20 \%$ no valor da coordenada Z, ocorreu um aumento na área da superfície de 6,7\%, ocorrendo um aumento no ganho térmico de $2 \%$. Já com a 
diminuição em $20 \%$ no valor da coordenada $Z$, a redução na área foi de $6,4 \%$, resultando numa diminuição no ganho térmico de 6,7\%.

\section{Figura 5 - Otimização do ganho térmico por alteração da forma}
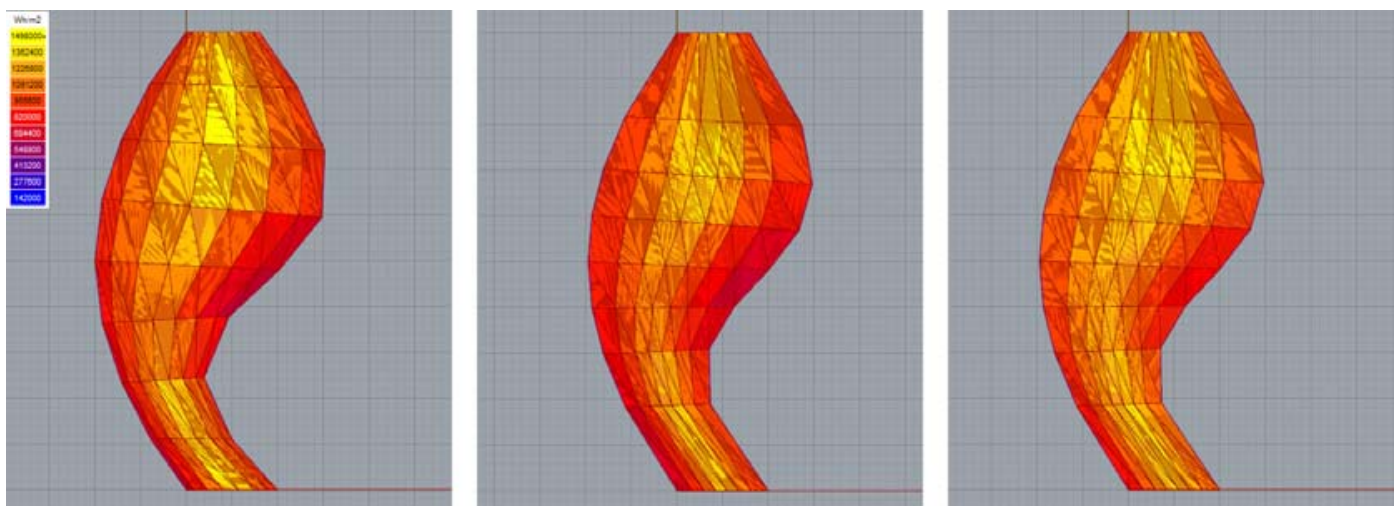

Fonte: própria

Na figura acima (Figura 5), nota-se como a modificação nas inclinações das faces alterou a insolação incidente. Essa alteração foi responsável por, mesmo com um aumento de $6,7 \%$ na área da superfície, um aumento do ganho térmico de somente $2 \%$.

Na aplicação de painéis foi utilizado o ganho térmico de cada subsuperfície. É importante ressaltar que o ganho térmico é diretamente proporcional à área e à insolação da face, não correspondendo à insolação somente. A aplicação dos painéis buscou alcançar $80 \%$ do ganho térmico da superfície original, aumentando a área de materiais opacos e isolantes.

Com uma gradação nas aberturas translúcidas das faces, as faces de baixo ganho térmico, mantiveram-se aberturas translúcidas maiores, permitindo a iluminação natural do ambiente interno, ao contrário das faces com alto ganho térmico, que tem aberturas menores ou nenhuma abertura (Figura 6). Se não houvesse esta gradação, com as mesmas aberturas em todas as faces, as faces ficariam subutilizadas, com excesso ou falta de material onde seria necessário para controlar a insolação incidente.

\section{Figura 6 - Otimização do ganho térmico pela aplicação de painéis}
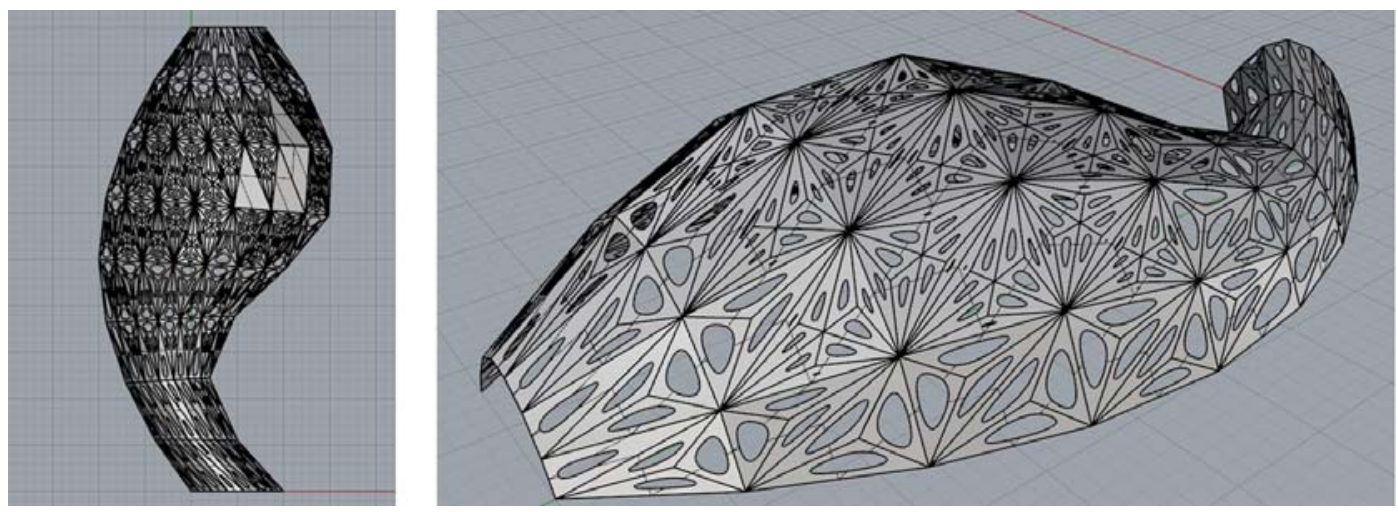

Fonte: própria 
Das três formas de otimização apresentadas, a utilização de painéis mostrou-se a mais adequada para a superfície testada, como se pode notar no Quadro 1 - Análises e Otimização, abaixo. Além do controle de ganho térmico, este método de otimização permite também o controle de iluminação natural no ambiente.

\section{Quadro 1 - Análises e Otimização}

\begin{tabular}{|c|c|c|c|}
\hline Superfície & $\begin{array}{c}\text { Ganho térmico } \\
\text { bruto (MWh) }\end{array}$ & $\begin{array}{c}\text { Ganho térmico } \\
\text { relativo (\%) }\end{array}$ & $\begin{array}{c}\text { Área translúcida } \\
\text { relativa (\%) }\end{array}$ \\
\hline Original - sem modificações & 1484 & 100 & $80 \%$ \\
\hline Rotação de 12 & 1486 & 100,1 & $80 \%$ \\
\hline Rotação de 95 & 1470 & 99 & $80 \%$ \\
\hline $\begin{array}{c}\text { Aumento das alturas relativas } \\
\text { em 20\% }\end{array}$ & 1514 & 102 & $80 \%$ \\
\hline $\begin{array}{c}\text { Redução das alturas relativas } \\
\text { em 20\% }\end{array}$ & 1386 & 93,3 & $80 \%$ \\
\hline Aplicação de painéis & 1223 & 82,4 & $25 \%$ \\
\hline
\end{tabular}

Fonte: própria

É importante ressaltar que, em outros casos, utilizando diferentes formas geométricas ou limitações, outras formas de otimização poderiam ser mais eficientes. A rotação, por exemplo, apresentou resultados muito semelhantes mesmo com as modificações por ter uma geometria com poucas curvaturas e sombreamento.

A alteração da forma é bastante interessante quando se tem liberdade para a modificação, não tendo outros parâmetros limitantes. Pela alteração das áreas e vetores normais das faces, pode-se alcançar ganhos térmicos significativamente mais baixos, modificando a forma para atender ao valor ideal.

Já o uso de painéis é muito interessante no caso de não se poder modificar a forma, trabalhando somente com as aberturas para maximizar ou minimizar os ganhos, bem como regular a iluminação natural.

\section{CONSIDERAÇÕES FINAIS}

Este experimento comprovou que a simples aplicação de uma regra prática bastante utilizada pelos arquitetos nas etapas iniciais do processo de projeto - a orientação da maior dimensão do edifício no sentido Norte-Sul - pode não ter efeitos significativos em relação ao ganho térmico total. O uso desse tipo de heurística, comum até alguns anos atrás, quando a realização de análises era um processo lento e complicado, pode não garantir a solução mais otimizada (Celani, 2012). O sistema aqui descrito, em que a manipulação da forma é acompanhada de uma resposta em tempo real sobre suas consequências em termos de ganho térmico, além da criação de regras que vinculam o dimensionamento de aberturas ao ganho solar local, resulta efetivamente em melhores soluções.

Como se pode ver no item anterior, as modificações aplicadas alteraram significativamente o ganho térmico do edifício. Apesar de algumas modificações apresentarem pouca variação em relação ao ganho térmico original, demonstram que é 
possível utilizar estas técnicas para adequar o ganho térmico do edifício ao valor desejado.

Pela modificação de parâmetros projetuais, foi possível otimizar o ganho térmico da superfície de diversos modos, demonstrando que o uso da parametrização é capaz de criar edifício mais 'inteligentes', com o auxílio de análises ambientais e simulações computacionais. Fornecendo resultados mais precisos e com menor gasto de tempo, é uma importante ferramenta no processo de projeto arquitetônico.

Outro aspecto interessante do uso da parametrização para a otimização, é que se pode pegar a mesma forma geométrica e mudar a sua localização geográfica, alterando o WeatherFile. Para cada local, a superfície seria otimizada de acordo com as necessidades locais, podendo adequar-se o ganho térmico. Ou ainda, no caso de estruturas modulares, pode-se variar a orientação solar da superfície e adequar seu ganho térmico com o uso dos painéis, garantindo em todos os módulos o mesmo ganho térmico independente de sua orientação.

Como próximo passo da pesquisa, serão revistas as formas de otimização, para identificar se, de fato, o uso de painéis é o mais adequado sempre. Serão também estudadas algumas combinações de formas de otimização, para alcançar melhores resultados. $\mathrm{O}$ último passo será desenvolver um método para gerar coberturas a partir da área a ser sombreada e o ganho térmico desejado.

\section{AGRADECIMENTOS}

À UNICAMP e ao LAPAC, pelo apoio recebido, e à FAPESP, pela bolsa de Iniciação Científica.

\section{REFERÊNCIAS}

BERG, M.; CHEONG, O.; KREVELD, M.; OVERMARS, M. Computational Geometry: Algorithms and Applications. $3^{\mathrm{a}}$ edição. Berlin: Springer, 2008.

BURRY, J.; BURRY, M. The New Mathematics of Architecture. Londres, Ed. Thames \& Hudson, 2010.

CELANI, G. Além da avaliação energética e ambiental nas etapas iniciais do processo de projeto. Parc, vol3, n2, p. 56-64, Outubro2012.

DIMCIC, M. What is your profession? Programming Architecture, 2013. Disponível em <http://www.youtube.com/watch?v=jwRPQrxxNDA> Acesso em: 26 abril 2014.

KOLAREVIC, B.; Architecture in the digital age: Design and manufacturing. $1^{\text {a }}$ Edição. Londres, Spon Press, 2005.

KOLAREVIC, B.; KLINGER, K. Manufacturing material effects: rethinking design and making in architecture. Nova York, Routledge, 2008. 